\title{
Luces y sombras en la predicción de coledocolitiasis: oportunidades para la investigación futura
}

\section{Lights and shadows in predicting choledocholithiasis: Opportunities for future research}

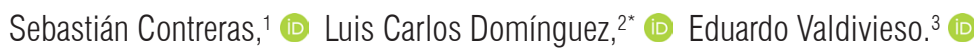

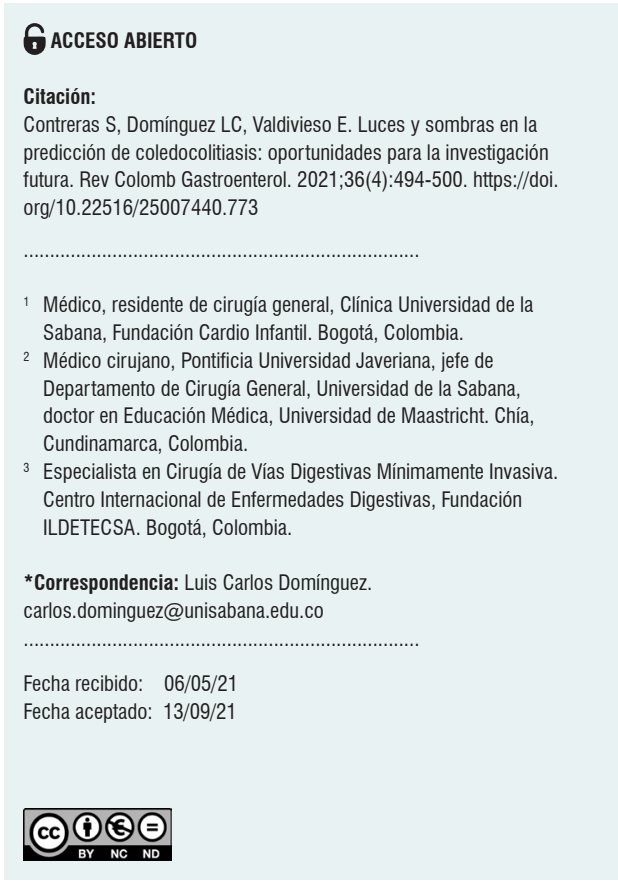

\begin{abstract}
Resumen
La coledocolitiasis secundaria es una entidad de alta prevalencia que involucra desafíos de diagnóstico y tratamiento; asimismo, genera un elevado uso de recursos y costo económico. Existen múltiples vacíos de conocimiento con respecto a los modelos de predicción clínica para el diagnóstico, estratificación en grupos de riesgo y manejo de pacientes con coledocolitiasis secundaria. Los principales vacíos recaen sobre su rendimiento diagnóstico, variables incluidas y umbrales de riesgo, así como sobre su costo-efectividad para el uso de recursos no invasivos e invasivos, y aplicación en grupos poblacionales especiales. Este artículo ahonda estos vacíos de conocimiento y propone una agenda que puede orientar la investigación futura.
\end{abstract}

\section{Palabras clave}

Coledocolitiasis, riesgo de coledocolitiasis, diagnóstico, modelo de predicción, umbrales.

\begin{abstract}
Secondary choledocholithiasis is a disease of high prevalence that involves diagnostic and treatment challenges; it implies a high use of resources and economic costs. There are significant knowledge gaps related to clinical prediction models, risk group classification, and patient treatment in secondary choledocholithiasis cases. Those gaps include diagnostic performance, variables, and risk thresholds, as well as cost-effectiveness for the use of non-invasive and invasive resources, and their application in special population groups. This study analyzes those knowledge gaps and outlines a guideline that could lead future research.
\end{abstract}

\section{Keywords}

Choledocholithiasis, Choledocholithiasis risk, Diagnostic, Prediction model, Thresholds.

\section{INTRODUCCIÓN}

La colelitiasis es una entidad quirúrgica frecuente a nivel global que afecta entre $10 \%$ y $15 \%$ de la población gene$\mathrm{ral}^{(1)}$. Una de sus complicaciones comunes es la coledocolitiasis, definida como la presencia de cálculos en la vía biliar común. Existen dos formas de presentación de coledocolitiasis: primaria y secundaria. Por una parte, la coledocolitiasis primaria se refiere a la formación in situ de cálculos en el colédoco; esta forma de presentación corresponde al $10 \%$ de los casos, y es más frecuente en países asiáticos ${ }^{(2)}$. Por otra parte, la forma secundaria ( $90 \%)$ ocurre por migración directa de cálculos desde la vesícula biliar. Este tipo de coledocolitiasis tiene una presentación variable, entre 4,6\% y $20,9 \%$ de los pacientes con colelitiasis en diferentes $\operatorname{contextos}^{(1,3-6)}$. La prevalencia y carga de enfermedad en Colombia se desconoce con exactitud, aunque algunos estudios estiman que cerca de $11,9 \%$ de los pacientes que ingresan a los servicios de urgencias con colecistitis aguda presenta coledocolitiasis ${ }^{(7)}$. 
La coledocolitiasis secundaria representa una gran carga económica para los sistemas de salud. En los Estados Unidos, por ejemplo, implica un costo aproximado de 6,6 billones de dólares anuales, incluido el costo de la colecistectomía como manejo definitivo ${ }^{(8)}$. Algunos estudios disponibles en Colombia informan sobre el costo de esta enfermedad para las instituciones, pero es poco lo que se conoce sobre su impacto económico para el sistema de salud ${ }^{(7,9,10)}$.

El diagnóstico y manejo de la coledocolitiasis secundaria ha sufrido grandes transformaciones en las últimas décadas, principalmente el uso de modelos de estratificación de riesgo desarrollados con base en la interacción de criterios clínicos, bioquímicos y de imágenes diagnósticas. De igual manera, la disponibilidad de pruebas diagnósticas no invasivas como la colangiografía por resonancia magnética nuclear (CRMN), la colangiografía por tomografía axial computarizada (TAC) y la endosonografía endoscópica (USE) han cambiado el abordaje diagnóstico de esta entidad, así como el refinamiento y aumento en el uso de técnicas endoscópicas para su tratamiento por colangiografía retrógrada endoscópica (CPRE), con respecto a procedimientos quirúrgicos que conllevan mayor morbilidad, estancia hospitalaria y costos de atención. Adicionalmente, los modelos de predicción y nuevas tecnologías no invasivas para el diagnóstico han disminuido las complicaciones de la CPRE con fines de diagnóstico, reportados hasta en el $10 \%{ }^{(11)}$.

Sin embargo, la predicción de coledocolitiasis secundaria permanece como un campo de permanente discusión debido a la persistencia de limitaciones propias de los modelos de predicción disponibles, entre las cuales vale la pena destacar:

- La interacción de las variables incluidas en los modelos.

- La validación de los modelos disponibles en diferentes contextos de práctica y poblaciones.

- Los criterios y mecanismos para la determinación de umbrales entre diferentes niveles de riesgo en los modelos disponibles.

- La aplicabilidad de modelos de predicción genéricos en poblaciones especiales.

- El impacto de los modelos de predicción en la costoefectividad del diagnóstico y tratamiento.

El objetivo de este estudio es exponer estos vacíos de conocimiento $y$ ofrecer potenciales preguntas para futuras investigaciones.

\section{VARIABLES INCLUIDAS EN LOS MODELOS DE PREDICCIÓN E INTERACCIÓN DE LAS MISMAS}

Tradicionalmente, el riesgo de coledocolitiasis se ha obtenido a partir de variables clínicas, perfil bioquímico hepático y hallazgos ecográficos. Las principales variables rela- cionadas con la presencia de cálculos en el colédoco, dada su fuerza de asociación, han sido la elevación de los niveles de bilirrubina, con puntos de corte reportados que varían entre 1,3 y $4,0 \mathrm{mg} / \mathrm{dL}$ (sensibilidad: $84 \%$, especificidad: $91 \%)^{(1,3,12-14)}$ y la dilatación de la vía biliar por ecografía > 6-8 mm (sensibilidad: $73 \%$; especificidad: $91 \%)^{(3)}$; así como también la elevación de otras pruebas bioquímicas como alanina-aminotransferasa (ALT), aspartato-aminotransferasa (AST), $\gamma$-glutamiltransferasa (GGT) y fosfatasa alcalina (FA), las cuales ofrecen una sensibilidad y especificidad aisladas de $81 \%$ y $25 \%$, respectivamente ${ }^{(3,14,15)}$. Finalmente, algunas variables de presentación clínica han sido incluidas en el abordaje diagnóstico, como la edad del paciente mayor de 55 años (sensibilidad: $57 \%$, especificidad: $49 \%$ ), pancreatitis biliar (sensibilidad: $7 \%-22,3 \%$; especificidad: $75 \%$ ), colangitis (sensibilidad: $21,7 \%$; especificidad: $99 \%)^{(1,11)}$ e ictericia (sensibilidad: $39 \%$, especificidad: $92 \%)$ como factores de riesgo aislados ${ }^{(15,16)}$.

A partir de esas variables, se han desarrollado diferentes modelos de predicción de coledocolitiasis en pacientes con colelitiasis. La mayoría de los modelos se deriva a partir de métodos estadísticos que incluyen regresiones logísticas multivariadas, con base en factores univariados identificados en estudios previos. Los modelos de predicción tienen como fin estratificar a los pacientes en diferentes grupos de riesgo sobre los cuales se determina la necesidad de métodos diagnósticos y estrategias de tratamiento ${ }^{(17,18)}$. Algunos modelos categorizan al paciente en dos grupos de riesgo (bajo y alto) ${ }^{(19)}$, otros en tres grupos (bajo, medio $y$ alto) $)^{(3,5,20)}$ y otros en subgrupos más específicos (bajo, medio-bajo, medio-alto, alto) $)^{(7,12,21,22)}$. Algunos ejemplos de este tipo de modelos son el de la Sociedad Americana de Gastroenterología y Endoscopia (SAGES) ${ }^{(5,20)}$ y el de la Sociedad Europea de Gastroenterología y Endoscopia $(\text { ESGE) })^{(3)}$

El modelo desarrollado por SAGES demuestra un rendimiento diagnóstico cercano al $70 \%$ en su versión de 2010 y es frecuentemente utilizado por cirujanos y gastroenterólogos a nivel mundial ${ }^{(23)}$. Sin embargo, ha sido ampliamente criticado debido principalmente a la ausencia de datos estadísticos que permitan su validación sobre muestras de población significativas en escenarios clínicos, lo cual limita los datos expuestos a análisis de características operativas definidas en estudios previos y opiniones de expertos. Algunos estudios han buscado la validación de este modelo en diferentes contextos ${ }^{(1,7,11,18,23)}$ y su comparación con modelos previos ${ }^{(15,16,24)}$. Sin embargo, sus resultados son heterogéneos y no permiten un análisis adecuado de la información, y en el momento no existen metaanálisis de los estudios disponibles que permitan generar recomendaciones sólidas sobre la aplicabilidad de este modelo de manera general. 
El modelo de la ESGE también es ampliamente utilizado a nivel mundial. Su desarrollo fue similar al de la SAGES, con base en revisiones de literatura y consenso de expertos. El modelo incluye variables bioquímicas, clínicas y ecográficas para la estratificación de tres grupos de riesgo (alto, medio y bajo $)^{(3)}$. Las características operativas del modelo son superiores al de SAGES en algunos estudios (sensibilidad: $89,57 \%$; especificidad: $96,24 \%)^{(25)}$; no obstante, dichas características operativas en grupos específicos, como el de los pacientes con colecistitis aguda, han demostrado bajo rendimiento diagnóstico con sensibilidad cercana al $49,2 \%$ y especificidad de $87,3 \%{ }^{(26)}$, lo que plantea los mismos retos de validación que presenta SAGES.

Finalmente, modelos alternativos han buscado equilibrar el peso relativo de cada variable y su interacción para la generación de nuevos modelos, y se han obtenido resultados diversos. Algunos modelos demuestran características operativas superiores al modelo de la SAGES ${ }^{(11,12)}$, mientras que otros demuestran características inferiores ${ }^{(13,22)}$. Por ejemplo, un estudio realizado en Colombia demostró un valor de 0,87 en las características operativas de receptor $(\mathrm{ROC})^{(7)}$, congruente con resultados en su validación externa ${ }^{(12)}$. Otros estudios realizados en Lituania y Arabia Saudí informan sobre hallazgos similares ${ }^{(13,22)}$. Nuevas propuestas especialmente en el último año (2021) han buscado mejorar la certeza en la predicción diagnóstica. Chisholm y colaboradores introdujeron un modelo en 2020 con base en un análisis multivariado en un centro de referencia especializado, con el objetivo de disminuir la cantidad de falsos positivos (pacientes llevados a CPRE no terapéutica) y falsos negativos (pacientes llevados a colecistectomía con coledocolitiasis $)^{(19)}$. Se obtuvo una adecuada discriminación de aproximadamente el $80 \%$ de la población, con tasas de CPRE fallida de $22 \%$ frente a $42 \%$ sobre SAGES para el grupo de riesgo alto, con una presentación de falsos negativos de 1,4\% para el riesgo bajo. Hubo un $20 \%$ de la población sobre el grupo intermedio que requirió estudios diagnósticos adicionales.

Con base en las limitaciones mencionadas, el desarrollo de nuevos estudios puede enfocarse en:

- Evaluar la interacción de las variables dentro del modelo de SAGES de forma objetiva. Los estudios de este tipo pueden contribuir a determinar, con base en métodos estadísticos, cuáles son los mejores puntos de corte para cada variable y su peso relativo dentro del modelo. Este tipo de análisis puede informar sobre características operativas del modelo con base en estudios empíricos originales.

- Validar los modelos disponibles en poblaciones diferentes. Existe la necesidad latente de validar el modelo de la SAGES y de la ESGE dado su uso creciente y amplia aceptación en diferentes contextos de práctica.
- Comparar el rendimiento operativo de los modelos, en términos de sus características operativas.

- Determinar el rendimiento de los modelos en situaciones clínicas que pueden afectar el comportamiento de las variables, como en casos de colecistitis aguda o edad avanzada, situaciones que han demostrado tener la capacidad de afectar los parámetros de los marcadores bioquímicos o el diámetro normal aceptado de la vía biliar, respectivamente.

\section{CRITERIOS Y MECANISMOS PARA LA DETERMINACIÓN DE UMBRALES DE RIESGO EN LOS MODELOS DISPONIBLES}

El problema del ajuste de los umbrales de riesgo dentro de los modelos radica en definir qué paciente entra en el grupo de alto, medio o bajo riesgo. Entonces, por ejemplo, mientras más estrictos sean los criterios de alto o bajo riesgo, menos pacientes serán incluidos en estos grupos, afectando la cantidad de pacientes en la zona de incertidumbre o de riesgo medio. La definición de los umbrales, por ende, puede impactar en el uso de recursos de diagnóstico y tratamiento. En este contexto, una adecuada discriminación de los umbrales de riesgo debe buscar disminuir la cantidad de pacientes llevados a CPRE no terapéutica, dada la carga de morbilidad del procedimiento; y distinguir de manera certera los pacientes que requieran estudios diagnósticos adicionales de los que pueden ser llevados a cirugía de manera segura. Estos modelos deben tener la capacidad de adaptación a los contextos propios de cada escenario clínico, teniendo en cuenta sus recursos disponibles, experiencia, recursos terapéuticos y costo-efectividad.

De manera general, los modelos de predicción propuestos muestran adecuadas características operativas globales en la discriminación de los grupos de riesgo. El modelo de la SAGES, por su parte, ha mostrado una sensibilidad diagnóstica en el grupo de riesgo alto de un $90 \%$ para la detección de coledocolitiasis, mientras que para el grupo de riesgo bajo se identifica una presentación cercana al 5 $\%^{(1,3,5,10)}$. Las validaciones nacionales han mostrado datos similares ${ }^{(23)}$; sin embargo, los grupos de riesgo intermedio persisten como interrogantes debido a la baja sensibilidad de diagnóstico, entre un $26 \%$ y un $49,5 \%^{(1,18,23)}$, y la cantidad de pacientes en este grupo bajo la distribución de riesgo actual hace que la mayoría de los pacientes deba ir a estudios diagnósticos prequirúrgicos adicionales, con resultados no conocidos en los desenlaces, que van desde el aumento inmediato en los costos de atención, retraso en el manejo terapéutico y aumento de complicaciones derivadas de una mayor estancia hospitalaria ${ }^{(27)}$.

Los umbrales, por tanto, afectan la funcionalidad global del modelo y uso de pruebas diagnósticas, sobre todo en 
los grupos de riesgo intermedio y bajo. Estos aspectos son importantes cuando se deciden los métodos diagnósticos, especialmente los no invasivos. Estos últimos, específicamente USE y CRMN, demuestran una sensibilidad de $97 \%$ y $87 \%$, y especificidad de $90 \%$ y $92 \%$, respectivamente ${ }^{(28)}$. Actualmente, son pruebas diagnósticas de uso generalizado, especialmente en el grupo de riesgo medio o de zona de incertidumbre, en el cual los métodos invasivos como la CPRE no se justifican por sus riesgos asociados (5\%-10 $\%)^{(15)}$. Al respecto, ensayos clínicos han demostrado que la USE y CRMN presentan un rendimiento comparable al de la CPRE para el diagnóstico de coledocolitiasis ${ }^{(29,30)}$; no obstante, aunque la USE presenta una sensibilidad ligeramente mayor para el diagnóstico en comparación con la $\mathrm{CRMN}^{(31)}$, su aplicabilidad se encuentra limitada por su baja disponibilidad y por ser una prueba de tipo dependiente del operador ${ }^{(28,32)}$.

Pero no solamente en el riesgo intermedio puede impactar el desempeño de métodos de precisión diagnóstica como la USE y la CRMN. En el riesgo alto, se asume una probabilidad que no es insignificante de un falso positivo, que se reporta sobre los modelos actuales hasta en un 50 $\%(27)$, hecho que generaría la indicación de un procedimiento invasivo asociado con riesgo como la CPRE con fines no terapéuticos. Es por esto que muchas escuelas en la actualidad consideran pertinente en determinadas situaciones, como un riesgo mayor de pancreatitis pos-CPRE, embarazo, o simplemente necesidad de llevar a cero la posibilidad de una CPRE no terapéutica, asumir el costo de confirmar la probabilidad alta de coledocolitiasis determinada por los modelos de predicción, con métodos diagnósticos como como la USE. ¿Qué niveles se están dispuestos a asumir como CPRE no terapéutica? y iqué porcentaje de pacientes llevados a cirugía con coledocolitiasis es adecuado?, persisten como interrogantes y deben ser ajustados a cada contexto clínico.

Con base en las limitaciones mencionadas, nuevos estudios pueden enfocarse en:

- Adaptar los umbrales de riesgo de acuerdo con el riesgo-beneficio de diagnosticar a todos los pacientes con el fin de disminuir la cantidad de falsos positivos en el riesgo alto y aumentar el porcentaje de pacientes que puede ser llevado de manera segura a cirugía sin requerimiento de estudios diagnósticos adicionales.

- Dinamizar los umbrales de riesgo del modelo de la SAGES y evaluar su impacto en el diagnóstico clínico sobre grupos poblacionales específicos, dando alternativas de ajuste a los contextos clínicos propios.

La necesidad de estudios clínicos prospectivos que permitan la validación de los cambios sobre las estructuras actuales, confirmación y dinámica de los modelos sobre escenarios clínicos propios se vuelven una necesidad en el desarrollo de esta temática y el cierre de brechas de conocimiento.

\section{APLICABILIDAD DE MODELOS DE PREDICCIÓN GENERICOS EN POBLACIONES ESPECIALES}

Otro punto importante sobre el cual existen limitaciones en la aproximación de los modelos de riesgo actual tiene que ver con su aplicación a grupos especiales de pacientes: adultos mayores (> 65 años), antecedente de colecistectomía, coledocolitiasis primaria, obstrucción biliar de origen maligno $\mathrm{y}$ aquellos con pancreatitis de origen biliar. En estos grupos de manera rutinaria se han aplicado las escalas de predicción diagnóstica diseñadas para pacientes con coledocolitiasis secundaria. No obstante, la validación de los modelos disponibles en estos grupos especiales es limitada. La adaptación de modelos de predicción a grupos para los cuales no han sido desarrollados ni validados puede afectar sus características operativas, en términos de falsos negativos y positivos, afectando el uso de recursos y pruebas diagnósticas. Por ejemplo, la diferenciación de la obstrucción biliar por malignidad versus enfermedad benigna no es sencilla. Múltiples predictores clínicos y de laboratorio se alteran en ambas entidades, incluso con el uso de elementos diagnósticos invasivos como la CPRE y el EUS. La determinación diferencial de riesgo es necesaria, principalmente la identificación de colestasis extra hepática por malignidad biliopancreática versus por patología biliar benigna ${ }^{(33)}$.

Los pacientes mayores de 65 años también se encuentran en esta categoría. Por una parte, este grupo de pacientes, cada vez mayor debido a la tasa de envejecimiento de la población, presenta características especiales dentro de las cuales se destacan una mayor prevalencia de colelitiasis, riesgo aumentado de presentar complicaciones y cambios fisiopatológicos en la generación de cálculos ${ }^{(34)}$. Estos factores hacen necesario el ajuste de los modelos genéricos a cada grupo de pacientes con el fin de proveer un tratamiento seguro y eficaz y disminuir el riesgo de complicaciones asociadas ${ }^{(32,35)}$. Por otra parte, la información y su uso en coledocolitiasis primaria es limitada. Algunos modelos en coledocolitiasis secundaria se han adaptado al abordaje de la coledocolitiasis primaria, pero su rendimiento es subóptimo al tratarse de entidades con una vía fisiopatológica diferente con alta tasa de recurrencia (hasta un $41,7 \%)^{(36)}$.

Con base en las limitaciones mencionadas, los nuevos estudios pueden enfocarse en adaptar y evaluar la validez predictiva de los modelos de predicción de coledocolitiasis secundaria en estos grupos especiales o en generar nuevos modelos específicos a partir de estudios empíricos en poblaciones especiales. 


\section{IMPACTO DE LOS MODELOS DE PREDICCIÓN EN LA COSTO-EFECTIVIDAD DEL DIAGNÓSTICO Y TRATAMIENTO}

En cuanto a costo-efectividad del diagnóstico, aún continua vigente la discusión sobre el impacto de los modelos en la costo-efectividad derivada del uso de pruebas diagnósticas no invasivas (CRMN y USE) e invasivas (CPRE). Los análisis de costo-efectividad han demostrado que el uso rutinario de CRMN o USE en pacientes con riesgo intermedio disminuye los costos de atención relacionados con la cantidad de CPRE no terapéuticas y sus complicaciones ${ }^{(37,38)}$. Así mismo, la comparación acerca de la utilidad de las pruebas no invasivas sugiere que la USE ofrece un mejor rendimiento diagnóstico; sin embargo, al incluir los costos relacionados con el procedimiento y compararlos con los de la CRMN, esta última parece ser más costo-efectiva ${ }^{(37)}$.

La aproximación a la costo-efectividad de la estratificación de riesgo es diversa. Kang y colaboradores demostraron la efectividad del modelo de SAGES en población mayor de 55 años, comparado con el uso rutinario de CRMN en todos los pacientes con sospecha de obstrucción biliar, incluida obstrucción maligna ${ }^{(39)}$. Sin embargo, sus resultados han sido revaluados por fallas en el análisis de sensibilidad del modelo y uso del modelo en diferentes tipos de población ${ }^{(40)}$. Otras aproximaciones a la costo-efectividad en estudios locales han demostrado rendimiento subóptimo del modelo de la SAGES con respecto a modelos nacionales ${ }^{(7,12)}$; sin embargo, los datos se encuentran limitados al impacto sobre el costo inmediato para las instituciones y carecen de evaluaciones a nivel del sistema de salud.

Adicionalmente, como objetivo secundario de algunos ensayos clínicos, se ha evaluado la costo-efectividad del manejo endoscópico en dos tiempos (CPRE + colecistectomía laparoscópica diferida) en comparación con el manejo operatorio en un solo tiempo (Rendezvous). Este último parece ofrecer mayor costo-efectividad ${ }^{(41)}$. Sin embargo, no todos los equipos quirúrgicos tienen el entrenamiento e instrumental necesario para su realización, lo cual ha sido evidenciado en estudios locales ${ }^{(10)}$. Adicionalmente, la mayor parte de estudios demuestra limitaciones en cuanto a la adaptación de los modelos en función de diferentes sistemas de aseguramiento y disponibilidad de recursos. Esta limitación tiene implicaciones para la atención oportuna, necesidad de referencia de pacientes a instituciones con mayor nivel de complejidad para la realización de estudios diagnósticos (especialmente CRMN y USE) y para el tratamiento definitivo. Estos aspectos aún requieren ser analizados en nuevos estudios para determinar los costos indirectos que se derivan de la aplicación de los modelos de atención en contextos con recursos limitados.

Con base en las limitaciones identificadas en literatura disponible, con respecto a la costo-efectividad de los modelos de predicción para el diagnóstico y tratamiento de la coledocolitiasis secundaria, los nuevos estudios pueden enfocarse en evaluar:

- La costo-efectividad y el impacto económico de los modelos para las instituciones y sistemas de salud.

- Cuál modelo ofrece mejor costo-efectividad en diferentes contextos de práctica.

- La adherencia de los profesionales al uso de los modelos y su impacto sobre la costo-efectividad.

- El impacto clínico relacionado con los tiempos de espera prolongados para la realización de pruebas diagnósticas no invasivas y la necesidad de referencia, así como el impacto económico para el sistema de salud y la institución derivado de estas demoras.

La realización de estos objetivos debe ser estudiada sobre muestras poblacionales significativas que permitan extrapolar el análisis de costo-efectividad sobre el diagnóstico, preferiblemente de bases de datos nacionales o de sistemas de aseguramiento, los cuales pueden ir de la mano de la viabilidad del desarrollo de estudios clínicos como objetivos de análisis secundario.

\section{CONCLUSIÓN}

La predicción de coledocolitiasis continúa siendo controversial. Múltiples aspectos han revolucionado la determinación de riesgo como el refinamiento en las técnicas endoscópicas con fines diagnósticos y terapéuticos, y el desarrollo de métodos de diagnóstico no invasivos como la USE y CRMN. Sin embargo, es imperativo identificar los vacíos de conocimiento en la literatura científica en torno a este tema, que pueden contribuir a mejorar los modelos de predicción disponibles. Aún es necesario evaluar las variables incluidas en los modelos de predicción, como su peso relativo e interacción. También es necesario que los modelos disponibles sean validados externamente en diferentes contextos y poblaciones. Igualmente, se requieren estudios enfocados en disminuir la incertidumbre diagnóstica relacionada con la determinación de los umbrales de riesgo y su aplicación en grupos especiales. Finalmente, es necesario profundizar en el impacto económico de los modelos para el sistema de salud y las instituciones. 


\section{REFERENCIAS}

1. Sethi S, Wang F, Korson AS, Krishnan S, Berzin TM, Chuttani R, Pleskow DK, Sawhney MS. Prospective assessment of consensus criteria for evaluation of patients with suspected choledocholithiasis. Dig Endosc. 2016;28(1):75-82.

https://doi.org/10.1111/den.12506

2. Jarnagin W. Blumgart's Surgery of the Liver, Biliary Tract and Pancreas. 6. ${ }^{\mathrm{a}}$ edition. Elsevier; 2016.

3. Manes G, Paspatis G, Aabakken L, Anderloni A, Arvanitakis M, Ah-Soune P, Barthet M, Domagk D, Dumonceau JM, Gigot JF, Hritz I, Karamanolis G, Laghi A, Mariani A, Paraskeva K, Pohl J, Ponchon T, Swahn F, Ter Steege RWF, Tringali A, Vezakis A, Williams EJ, van Hooft JE. Endoscopic management of common bile duct stones: European Society of Gastrointestinal Endoscopy (ESGE) guideline. Endoscopy. 2019;51(5):472-491. https://doi.org/10.1055/a-0862-0346

4. Norero E, Norero B, Huete A, Pimentel F, Cruz F, Ibáñez L, Martínez J, Jarufe N. Rendimiento de la colangiografía por resonancia magnética en el diagnóstico de coledocolitiasis. Rev Med Chil. 2008;136(5):600-5.

5. ASGE Standards of Practice Committee, Buxbaum JL, Abbas Fehmi SM, Sultan S, Fishman DS, Qumseya BJ, Cortessis VK, Schilperoort H, Kysh L, Matsuoka L, Yachimski P, Agrawal D, Gurudu SR, Jamil LH, Jue TL, Khashab MA, Law JK, Lee JK, Naveed M, Sawhney MS, Thosani N, Yang J, Wani SB. ASGE guideline on the role of endoscopy in the evaluation and management of choledocholithiasis. Gastrointest Endosc. 2019;89(6):1075-1105.e15. https://doi.org/10.1016/j.gie.2018.10.001

6. Collins C, Maguire D, Ireland A, Fitzgerald E, O'Sullivan GC. A prospective study of common bile duct calculi in patients undergoing laparoscopic cholecystectomy: natural history of choledocholithiasis revisited. Ann Surg. 2004;239(1):28-33. https://doi.org/10.1097/01.sla.0000103069.00170.9c

7. Domínguez Torres LC, Niño Puentes NA, Rubio Vermeo OD, Valdivieso Rueda E, Rugeles Quintero SJ. Sanabria Á. Desarrollo de un modelo para la predicción de coledocolitiasis: análisis e impacto de la discriminación de riesgo en el uso de recursos. Medicina (B Aires). 2011;33(3):160-75.

8. Hall MJ, Schwartzman A, Zhang J, Liu X. Ambulatory Surgery Data From Hospitals and Ambulatory Surgery Centers: United States, 2010. Natl Health Stat Report. 2017;(102):1-15.

9. Angel A, Rosero G, Crispín M, Valencia J, Muñoz A, Cadavid A. Coledocolitiasis [internet]. Guías de Manejo en Cirugía; 2018 [consultado 15 de junio de 2015]. Disponible en: https://www.ascolcirugia.org/images/ resources/PDF/guiasCirugia/coledocolitiasis.pdf

10. Acebedo B, Almeyda M, Chaves K, Higuera E. Análisis de costo efectividad del Rendez vous laparoendoscópico biliar versus el manejo convencional en dos tiempos para el tratamiento de la colecistocoledocolitiasis en la clínica Foscal de Bucaramanga - Colombia. Universidad Autónoma de Bucaramanga; 2014.

11. Kang J, Paik KH, Lee JC, Kim HW, Lee J, Hwang JH, Kim J. The Efficacy of Clinical Predictors for Patients with Intermediate Risk of Choledocholithiasis. Digestion. 2016;94(2):100-105. https://doi.org/10.1159/000448917

12. Henao-Ardila J, Londoño SO, Monsalve SC, Quintero VM, Sanabria Á. Validación externa del modelo HUSI para la predicción de coledocolitiasis en pacientes con colelitiasiscolecistitis. Rev Colomb Cirugía. 2018;33(2):135-44. https://doi.org/10.30944/20117582.55

13. Aleknaite A, Simutis G, Stanaitis J, Valantinas J, Strupas K. Risk assessment of choledocholithiasis prior to laparoscopic cholecystectomy and its management options. United European Gastroenterol J. 2018;6(3):428-438. https://doi.org/10.1177/2050640617731262

14. Halabi WJ, Jafari MD, Kang CY, Nguyen VQ Carmichael JC, Mills S, Pigazzi A, Stamos MJ. Colonic volvulus in the United States: trends, outcomes, and predictors of mortality. Ann Surg. 2014;259(2):293-301. https://doi.org/10.1097/SLA.0b013e31828c88ac

15. Abboud PA, Malet PF, Berlin JA, Staroscik R, Cabana MD, Clarke JR, Shea JA, Schwartz JS, Williams SV. Predictors of common bile duct stones prior to cholecystectomy: a metaanalysis. Gastrointest Endosc. 1996;44(4):450-5. https://doi.org/10.1016/s0016-5107(96)70098-6

16. Menezes N, Marson LP, debeaux AC, Muir IM, Auld CD. Prospective analysis of a scoring system to predict choledocholithiasis. Br J Surg. 2000;87(9):1176-81. https://doi.org/10.1046/j.1365-2168.2000.01511.x

17. Costi R, Gnocchi A, Di Mario F, Sarli L. Diagnosis and management of choledocholithiasis in the golden age of imaging, endoscopy and laparoscopy. World J Gastroenterol. 2014;20(37):13382-401. https://doi.org/10.3748/wjg.v20.i37.13382

18. Suarez AL, LaBarre NT, Cotton PB, Payne KM, Coté GA, Elmunzer BJ. An assessment of existing risk stratification guidelines for the evaluation of patients with suspected choledocholithiasis. Surg Endosc. 2016;30(10):4613-8. https://doi.org/10.1007/s00464-016-4799-8

19. Chisholm PR, Patel AH, Law RJ, Schulman AR, Bedi AO, Kwon RS, Wamsteker EJ, Anderson MA, Elta GH, Govani SM, Prabhu A. Preoperative predictors of choledocholithiasis in patients presenting with acute calculous cholecystitis. Gastrointest Endosc. 2019;89(5):977-983.e2. https://doi.org/10.1016/j.gie.2018.11.017

20. Narula VK, Fung EC, Overby DW, Richardson W, Stefanidis D; SAGES Guidelines Committee. Clinical spotlight review for the management of choledocholithiasis. Surg Endosc. 2020;34(4):1482-1491. https://doi.org/10.1007/s00464-020-07462-2

21. El Mouzan MI, Al Mofleh IA. The Saudi Journal of Gastroenterology. Saudi J Gastroenterol. 1995;1(2):73-4. 
22. Al-Jiffry BO, Khayat S, Abdeen E, Hussain T, Yassin M. A scoring system for the prediction of choledocholithiasis: a prospective cohort study. Ann Saudi Med. 2016;36(1):57-63. https://doi.org/10.5144/0256-4947.2016.57

23. Toro Calle J, Guzmán Arango C, Ramírez Ceballos M, Guzmán Arango N. ¿Son los criterios de la ASGE suficientes para la estratificación del riesgo de coledocolitiasis? Rev Colomb Gastroenterol. 2020;35(3):304-10. https://doi.org/10.22516/25007440.464

24. Barkun AN, Barkun JS, Fried GM, Ghitulescu G, Steinmetz $\mathrm{O}$, Pham C, Meakins JL, Goresky CA. Useful predictors of bile duct stones in patients undergoing laparoscopic cholecystectomy. McGill Gallstone Treatment Group. Ann Surg. 1994;220(1):32-9. https://doi.org/10.1097/00000658-199407000-00006

25. Jagtap N, Hs Y, Tandan M, Basha J, Chavan R, Nabi Z, Kalapala R, Reddy PM, Ramchandani M, Gupta R, Lakhtakia S, Darishetty S, Rao GV, Reddy DN. Clinical utility of ESGE and ASGE guidelines for prediction of suspected choledocholithiasis in patients undergoing cholecystectomy. Endoscopy. 2020;52(7):569-573. https://doi.org/10.1055/a-1117-3451

26. Reddy S, Jagtap N, Kalapala R, Ramchandani M, Lakhtakia S, Basha J, Nabi Z, Karyampudi A, Chavan R, Tandan M, Gupta R, Reddy DN. Choledocholithiasis in acute calculous cholecystitis: guidelines and beyond. Ann Gastroenterol. 2021;34(2):247-252. https://doi.org/10.20524/aog.2020.0562

27. Ko C, Buxbaum J. Do the 2019 ASGE choledocholithiasis guidelines reduce diagnostic ERCP? Gastrointest Endosc. 2021 Jun;93(6):1360-1361. https://doi.org/10.1016/j.gie.2020.12.046

28. Giljaca V, Gurusamy KS, Takwoingi Y, Higgie D, Poropat G, Stimac D, Davidson BR. Endoscopic ultrasound versus magnetic resonance cholangiopancreatography for common bile duct stones. Cochrane Database Syst Rev. 2015;2015(2):CD011549. https: / doi.org/10.1002/14651858.CD011549

29. ASGE Standards of Practice Committee, Maple JT, BenMenachem T, Anderson MA, Appalaneni V, Banerjee S, Cash BD, Fisher L, Harrison ME, Fanelli RD, Fukami N, Ikenberry SO, Jain R, Khan K, Krinsky ML, Strohmeyer $\mathrm{L}$, Dominitz JA. The role of endoscopy in the evaluation of suspected choledocholithiasis. Gastrointest Endosc. 2010;71(1):1-9. https://doi.org/10.1016/j.gie.2009.09.041

30. Prat F, Amouyal G, Amouyal P, Pelletier G, Fritsch J, Choury A, Etienne JP. Prospective controlled study of endoscopic ultrasonography (EUS) and retrograde cholangiography (ERC) in patients with suspected common bile duct lithiasis (CBDL). Gastrointest Endosc. 1995;41(4):411.

31. Loureiro Mde P, Almeida RA, Claus CM, Bonin EA, CuryFilho AM, Dimbarre D, Costa MA, Vital ML. Laparoscopic resection of gastrointestinal stromal tumors (GIST). Arq Bras Cir Dig. 2016;29(1):1-4. https://doi.org/10.1590/0102-6720201600010001
32. Williams E, Beckingham I, El Sayed G, Gurusamy K, Sturgess R, Webster G, Young T. Updated guideline on the management of common bile duct stones (CBDS). Gut. 2017;66(5):765-782. https://doi.org/10.1136/gutjnl-2016-312317

33. Kasapidis P, Mironidis L, Giannakopoulos A, DElis V, Balastos V, Konstantinidis A, Skandalis N. Clinical discrimination between choledocholithiasis and biliopancreatic malignancy based on a new biochemical model. Ann Gastroenterol. 2008;21(2):119-26.

34. Rosenthal RA, Andersen DK. Surgery in the elderly: observations on the pathophysiology and treatment of cholelithiasis. Exp Gerontol. 1993;28(4-5):459-72. https://doi.org/10.1016/0531-5565(93)90071-k

35. Costi R, DiMauro D, Mazzeo A, Boselli AS, Contini S, Violi V, Roncoroni L, Sarli L. Routine laparoscopic cholecystectomy after endoscopic sphincterotomy for choledocholithiasis in octogenarians: is it worth the risk? Surg Endosc. 2007;21(1):41-7. https://doi.org/10.1007/s00464-006-0169-2

36. Zhang J, Ling X. Risk factors and management of primary choledocholithiasis: a systematic review. ANZ J Surg. 2021;91(4):530-536. https://doi.org/10.1111/ans.16211

37. Scheiman JM, Carlos RC, Barnett JL, Elta GH, Nostrant TT, Chey WD, Francis IR, Nandi PS. Can endoscopic ultrasound or magnetic resonance cholangiopancreatography replace ERCP in patients with suspected biliary disease? A prospective trial and cost analysis. Am J Gastroenterol. 2001;96(10):2900-4. https://doi.org/10.1111/j.1572-0241.2001.04245.x

38. Carlos RC, Scheiman JM, Hussain HK, Song JH, Francis IR, Fendrick AM. Making cost-effectiveness analyses clinically relevant: the effect of provider expertise and biliary disease prevalence on the economic comparison of alternative diagnostic strategies. Acad Radiol. 2003;10(6):620-30. https://doi.org/10.1016/s1076-6332(03)80080-6

39. Kang SK, Hoffman D, Ferket B, Kim MI, Braithwaite RS. Risk-stratified versus Non-Risk-stratified Diagnostic Testing for Management of Suspected Acute Biliary Obstruction: Comparative Effectiveness, Costs, and the Role of MR Cholangiopancreatography. Radiology. 2017;284(2):468-481. https://doi.org/10.1148/radiol.2017161714

40. Halpern EF, Pandharipande PV. Behind the Numbers: Sensitivity Analysis in Cost-Effectiveness Modeling. Radiology. 2017;284(2):310-312. https://doi.org/10.1148/radiol.2017170242

41. Bansal VK, Misra MC, Rajan K, Kilambi R, Kumar S, Krishna A, Kumar A, Pandav CS, Subramaniam R, Arora MK, Garg PK. Single-stage laparoscopic common bile duct exploration and cholecystectomy versus two-stage endoscopic stone extraction followed by laparoscopic cholecystectomy for patients with concomitant gallbladder stones and common bile duct stones: a randomized controlled trial. Surg Endosc. 2014;28(3):875-85. https://doi.org/10.1007/s00464-013-3237-4 